\title{
ERODIBILIDADE DOS SOLOS DO ESTADO DE SÃO PAULO*
}

\author{
O. FREIRE \\ J. E. S. Pessotti
}

\section{RESUMO}

\begin{abstract}
Fol calculado pelo método nomográfico de WISCHMDHIFR et al (1971), o valor dos indices de erodibilidade dos Grandes Grupos de Solos do Estado de São Paulo (COMISSÃO DE SOLOS, (1960).

Os valores encontrados variam de 0,02 a 0,67 . Pode-se afirmar que os Latossois são, de maneira geral, mais resistentes a erosăo dos que os solos podzólicos e que dentre os podzólicos a erodibilidado aumenta a medida que aumenta o gradiente textural entro os horizontes $\mathbf{A} \bullet \mathbf{B}$.
\end{abstract}

\section{INTRODUÇAO}

A erodibilidade é o fator mais importante para a escolha de práticas conservacionistas, além de representar a propriedade do solo que reflete o fato de que diferentes solos perdem quantidades variáveis de material, quando submetidos às mesmas condições de declividade, comprimento de rampa, manejo e chuva.

A influência da natureza do solo sobre a erodibilidade é obvia; sendo esta propriedade o produto da interação da profundidade, permeabilidade, textura, estrutura e pedregosidade.

Os solos do Estado de São Paulo são de natureza muito variável (1960); por esse motivo, pode-se esperar que seus valores de erodibilidade sejam, também, muito diferentes. Esse fato tem sido comprovado pela observação; entretanto, para o planejamento conservacionista, necessita-se dos valores numéricos dessa característica.

O objetivo deste trabalho é obter o índice de erodibilidade dos solos do Estado de São Paulo. Os resultados obtidos, além de representarem a

* Entregue para publicaç̃̃o em 04/12/1974.

* Departamento de Solos e Geologia - ESALQ. 
avaliação de uma característica dos solos que até hoje não mereceu a atenção dos pesquisadores, é o primeiro passo para o desenvolvimento de pesquisas conservacionistas de acordo com os princípios mais modernos e promissores.

\section{REVISAO DA LITERATURA}

WISCHMEIER e SMITH (1961) descreveram a erodibilidade do solo (K) como as perdas de solo em toneladas por hectare por unidade do fator chuva em condições padrão de declividade, comprimento de rampa, manejo e prática conservacionista. As condições padrão propostas por aqueles autores são as seguintes: declividade de $9 \%$, comprimento de rampa de $22 \mathrm{~m}$, ausência de vegetação e de práticas conservacionistas.

O fator chuva (EI) foi descrito por WISCHMEIER (1959) como o produto da energia cinética da chuva pela intensidade máxima em $30 \mathrm{mi}$ nutos.

Esses parâmetros foram combinados com os fatores declividade-comprimento do declive, manejo e práticas conservacionistas para compor a equação de perdas (WISCHMEIER e SMITH, 1961).

WISCHMEIER et al (1971) idealizaram um sistema nomográfico baseado em cinco parâmetros para a determinação dos valores de erodibilidade dos solos. Os parâmetros utilizados podem ser obtidos através das determinações de rotina feitas em laboratório e das descrições morfológicas dos perfis de solos.

Valores do índice de erodibilidade já foram obtidos para solos dos Estados Unidos (WISCHMEIER e SMITH, 1965) e para alguns solos tropicais (SMITH e ABRUNA, e FREIRE e PESSOTTI, 1955 e 1974).

Essas mesmas informações são necessárias para outros solos de outras regiões geográficas para que a predição da erosão e escolha do sistema de manejo e práticas conservacionistas possa ser calculada eficientemente para o Estado de São Paulo.

\section{MATERIAL E MÉTODO}

\section{Material}

O material que serviu de objeto para o estudo da erodibilidade dos solos do Estado de São Paulo é constituído pelos dados analíticos apresentados no Levantamento de Recolhimento dos Solos do Estado de São Paulo (1960).

\section{Mẻtodo}

O método nomográfico de WISCHMEIER et al (1971) interrelaciona graficamente cinco fatores para a determinação da erodibilidade do solo. 
Essa combinação foi obtida estatisticamente da análise de dados referentes a cinquenta e cinco solos submetidos a tratamentos com chuva artificial e mais treze solos submetidos a tratamentos com chuva natural, em experimentos conduzidos a longo prazo (1959).

Os parâmetros utilizados para predizer a erodibilidade do solo são os seguintes: porcentagem de limo mais porcentagem de areia muito fina, porcentagem de areia maior do que $0,1 \mathrm{~mm}$, porcentagem de matéria orgânica, estrutura e permeabilidade.

0 nomógrafo de Wischmeier está apresentado na figura 1.

0 processo para a predição do índice de erodibilidade do solo consiste em se aplicar, na escala da esquerda, o valor da porcentagem de limo mais a porcentagem de areia muito fina. Continua-se aplicando, ao nomógrafo, os demais parâmetros na seguinte sequência: porcentagem de areia maior do que $0,1 \mathrm{~mm}$, porcentagem de matéria orgânica, estrutura e permeabilidade.

As linhas pontilhadas marcadas, na figura 1, ilustram o processo para a determinação dos valores de $\mathrm{K}$.

Para o cálculo dos valores da erodibilidade de cada um dos Grandes Grupos de Solos do Estado de São Paulo utilizaram-se dados de pelo menos dois perfis da mesma unidade, exceto para o Podzol Hidromórfico e Litosois. 


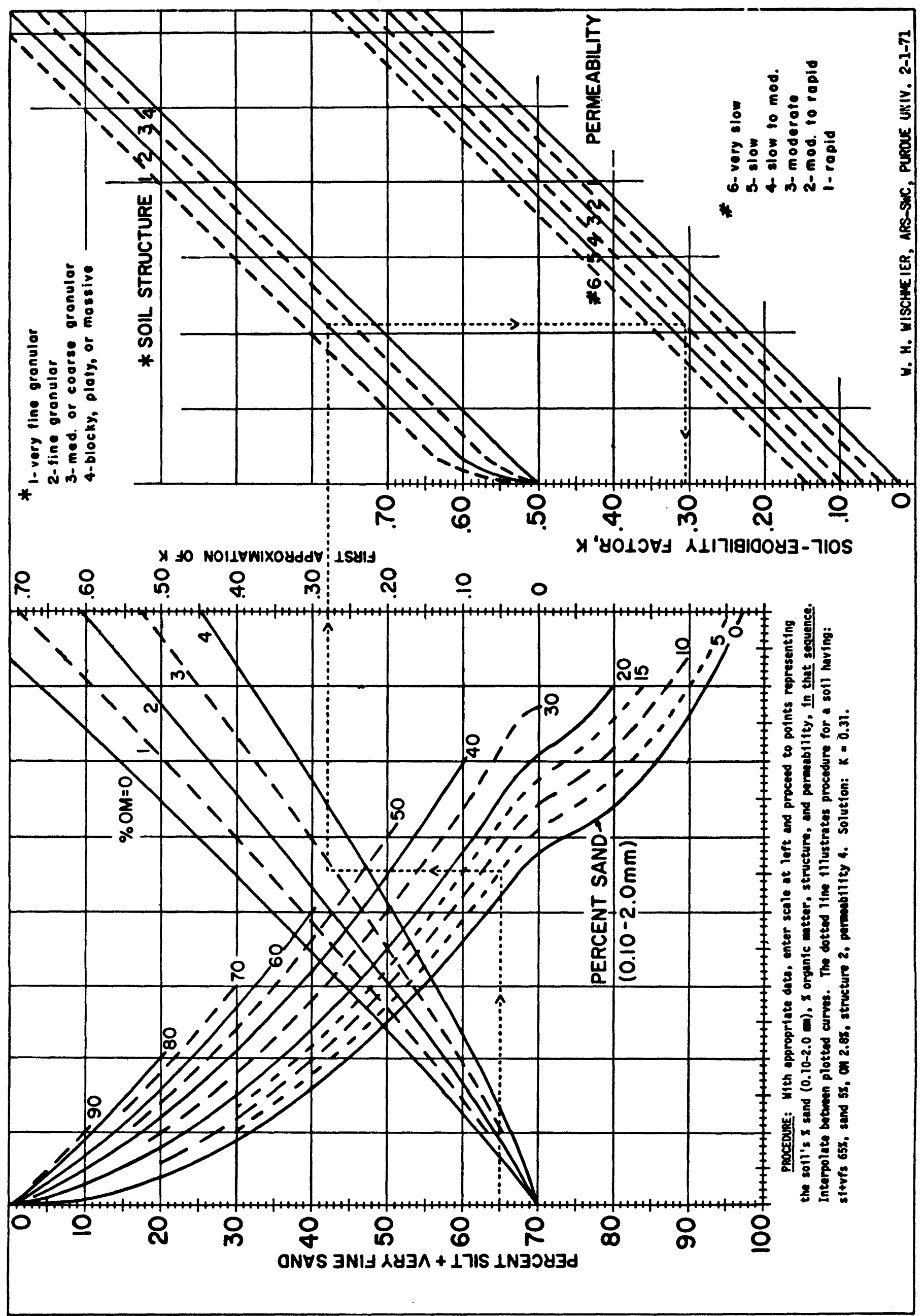


Com base na distribuição dos valores do índice de erodibilidade do horizonte superficial de todas as unidades, foram propostas cinco classes de erodibilidade, assim denominadas:

$$
\begin{aligned}
\text { muito alta : } & \mathrm{K}>0,50 ; \\
\text { alta }: & \mathrm{K}=0,50-0,35 ; \\
\text { média : } & \mathrm{K}=0,35-0,25 ; \\
\text { baixa : } & \mathrm{K}=0,25-0,10 \mathrm{e} \\
\text { muito baixa : } & \mathrm{K}<0,10 .
\end{aligned}
$$

\section{RESULTADOS E DISCUSSÃO}

Os dados de $\mathrm{K}$ obtidos pelo método nomográfico de WISCHMEIER et al (1971) para os solos do Estado de São Paulo estão apresentados no apêndice. Esses dados foram calculados para todos os Grandes Grupos que ocorrem no Estado de São Paulo, exceto para Alúvios e para as Associações de Solos. ciações de Solos.

De acordo com o critério proposto no ítem 4, os solos do Estado de São Paulo foram classificados quanto à erodibilidade, como está apresentado no quadro 1.

Pode-se observar que os horizontes B nem sempre apresentam um índice varia de Grande Grupo para Grande Grupo e de horizonte para horizonte nos perfis de um mesmo Grande Grupo.

Os valores mais altos encontrados referem-se ao horizonte $\mathrm{C}_{2}$ dos Solos de Campos do Jordão e à camada $R$ do Litosol fase Filito-Xisto; enquanto os valores mais baixos referem-se ao horizonte A da Terra Roxa Estruturada.

Pode-se observar que os horizontes $B$ nem sempre apresenta um índice de erodibilidade mais alto do que o dos horizontes A. Nos perfis de Mediterrânico Vermelho-Amarelo, Terra Roxa Estruturada, Latosol Roxo, Latosol Vermelho-Escuro fase arenosa e orto, Latosol Vermelho-Amarelo fase rasa, fase arenosa, fase terraço e «intergrade» para Podzólico Vermelho-Amarelo, assim como nos solos de Campos do Jordão, a erodibilidade aumenta com a profundidade. 
Quadro I: Classificação dos Solos do Estado de São Paulo segundo sua erodibilidade.

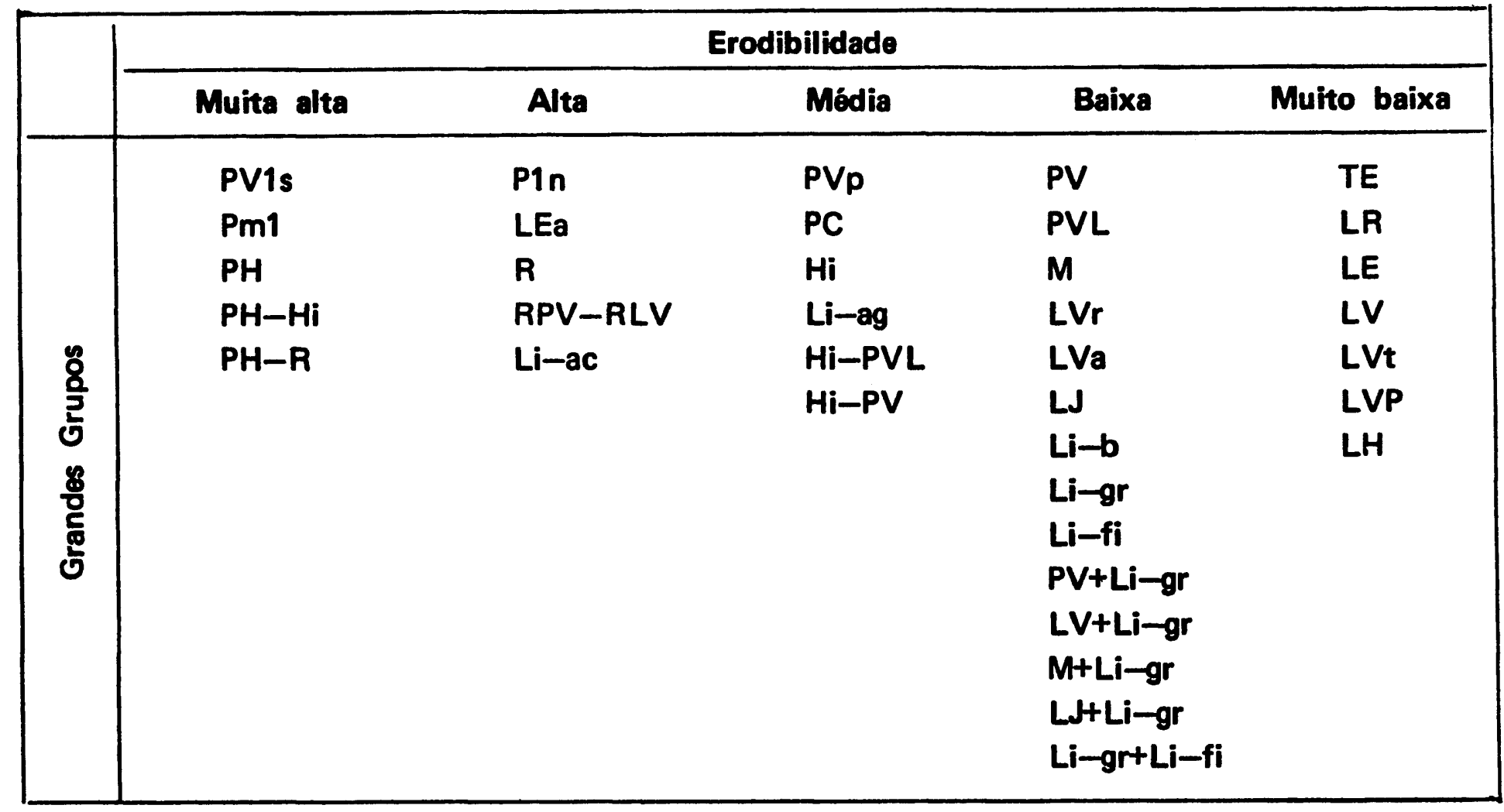

Apenas nos solos podzólicos a erodibilidade diminui com a profundidade até o horizonte $\mathrm{C}$, o qual geralmente apresenta valores de $\mathrm{K}$ semelhantes aos das camadas superficiais.

Desde que a erosão agrícola se processe nas camadas superficiais do solo, a consideração dos valores do índice de erodibilidade das camadas superficiais é de maior importância, salvo quando as camadas subjacentes estão expostas.

Sob esse aspecto, os Grandes Grupos de solos podzólicos são os que apresentam maiores valores de $\mathrm{K}$ para o horizonte superficial. Em seguida, correspondendo a valores altos de K, estão o Grande Grupo Regosol, Latosois Vermelho-Escuro arenoso e Litosol de Arenito Calcário.

Os valores de erobilidade média estão representados nos perfis dos Grandes Grupos dos Litosois de argilito e hidromórficos.

Os perfis que apresentam erodibilidade baixa são os dos Grandes Grupos Mediterrânico Vermelho-Amarelo, Latosois Vermelho-Amarelo, Litosois de basalto, granito e filito, além dos solos de Campos do Jordão.

Os solos mais resistentes à erosão são representados pelos perfis de Terra Roxa Estruturada, Latosol Roxo, Latosol Vermelho Escuro-orto, Latosol Vermeloh-Amarelo-orto, fase terraço «integrade» para Podzólico Vermelho-Amarelo e Latosol Vermelho-Amarelo Húmico.

Os Podzólicos apresentam valores de $\mathrm{K}$ muito variável. Os Podzólicos Vermelho-Amarelo variação Laras, Marília e o Podzol Hidromórfico apresentam valores muito alto para o índice K. O PVA variação Lins apresenta 
um valor alto; enquanto que o PVA variação Piracicaba e os Podzolizados com Cascalho apresentam valores médios.

O PVA-orto e o PVA «intergrade» para LVA apresentam valores baixos.

Deve-se notar que esses resultados numéricos correspondem sempre aos valores comparativos esperados em face da observação de campo.

\section{CONCLUSÓES}

De acordo com os resultados obtidos pelo método de WISCHMEIER et al (1971) para a predição da erodibilidade dos solos do Estado de São Paulo, pode-se concluir que:

1. os solos mais sujeitos à erosão são os pertencentes aos Grandes Grupos Podzólico Vermelho-Amarelo var. Laras, Marília e Lins, Podzol Hidromórfico, Regosol, Litosol de Arenito Calcário e LVE fase arenosa;

2. a erodibilidade dos solos podzólicos é tanto maior quanto maior é o gradiente textural entre os horizontes A e B;

3. os solos mais resistentes à erosão são a Terra Roxa Estruturada e todos os Latosois, exceto o LVA fase arenosa e fase rasa, além do LVE fase arenosa;

4. os valores da erodibilidade dos solos do Estado de São Paulo variam de 0,02 a 0,67 .

\section{SUMMARY}

ERODIBILITY OF SOME TROPICAL SOILS

Erodibility values for the soils of São Paulo State are reported in this paper. Wichmeier's nomograph method was used to determine the $K$ values.

The erodibility values are higher in the soils with an argilic horizon and lower in the soils with on oxic horizon or an oxic horizon associated with an argilic horizon.

The $K$ values ranged from 0,02 to 0,67 .

\section{LITERATURA CITADA}

COMISSAO DE SOLOS - Levantamento de Reconhecimento dos Solos do Estado de São Paulo. Rio de Janeiro, Centro Nacional de Ensino e Pesquisas Agronômicas, 1960 (Boletim $\mathrm{n}^{\circ}$ 12).

FREIRE, O. \& J. E. S. PESSOTTI - Erodibilidade dos Solos de Piracicaba. Rev. Agr. (em impressão).

RANZANI, G., O. FREIRE, T. KINJO - Carta de Solos do Município de Piracicaba - mimeografado. ESALQ-USP, 1966. 
SMITH, R. M. \& F. ABRUNA. - Soil and Water Conservation Research in Puerto Rico, 1938 to 1947. Univ. of Puerto Rico Agr. Exp. Sta. Bul. 125, May. 1955.

WISCHMEIER ,W. H. - A Rainfall Erosion Index for a Universal Soil-Loss Equation. Soil Sci. Soc. Amer. Proc. 23 : 246-249. 1959.

WISCHMEIER, W. H. \& D. D. SMITH. - A Universal Soil-Loss Estimating Equation to Guide Conservation Form Planning. $7^{\text {th }}$ Congr. Intern. Soil Scl. Soc. Trans. 1(2). 1961.

WISCHMEIER, W. H. \& D. D. SMITH. - Predicting Rainfall-Erosion Losses from Cropland East of the Rocky Mountains. Agric. Handbook n.॰ 282, USDA, ARS, 47 p., 1965.

WISCHMEIER, W. H., C. B. JOHNSON \& B. V. CROSS - A Soil Erodibility Nomograph for Farmland and Construction Sites. Journal of Soil and Wate Conservation $26(5): 189-193.1971$. 


\section{APENDICE}

Podzólico Venmelho-Amarelo - Orto

Perfil n. ${ }^{\circ} 1$

Perfil n.० 2

\begin{tabular}{crccrc} 
Horizonte & Espessura & $\mathrm{K}$ & Horizonte & Espessura & $\mathrm{K}$ \\
$\mathrm{A}_{1}$ & $0-10$ & 0,34 & $\mathrm{~A}_{1}$ & $0-10$ & 0,16 \\
$\mathrm{~A}_{2}$ & $10-25$ & 0,38 & $\mathrm{~A}_{2}$ & $10-30$ & 0,28 \\
$\mathrm{~B}_{22}$ & $25-80$ & 0,16 & $\mathrm{~A}_{3}$ & $30-50$ & 0,20 \\
$\mathrm{~B}_{23}$ & $80-100$ & 0,21 & $\mathrm{~B}_{1}$ & $50-75$ & 0,18 \\
$\mathrm{~B}_{3}$ & $100-130$ & 0,28 & $\mathrm{~B}_{2}$ & $75-118$ & 0,23 \\
$\mathrm{C}$ & $130-200$ & 0,59 & $\mathrm{~B}_{3}$ & $118-148$ & 0,40 \\
& & & $\mathrm{C}$ & $148-168$ & 0,43 \\
\hline
\end{tabular}

Podzólico Vermelho-Amarelo - var. Piracicaba

\begin{tabular}{cccccc} 
& Perfil n..$^{\circ}$ & \multicolumn{3}{c}{ Perfil n..$^{\circ}$} \\
Horizonte & Espessura & $\mathrm{K}$ & Horizonte & Espessura & $\mathrm{K}$ \\
$\mathrm{Ap}$ & 0,23 & 0,36 & $\mathrm{Ap}$ & $0-20$ & 0,28 \\
$\mathrm{~B}_{1}$ & $23-44$ & 0,17 & $\mathrm{~B}_{22}$ & $20-50$ & 0,11 \\
$\mathrm{~B}_{2}$ & $44-58$ & 0,14 & $\mathrm{~B}_{23}$ & $50-100$ & 0,12 \\
$\mathrm{~B}_{22}$ & $58-150$ & 0,12 & $\mathrm{C}$ & $100-140$ & 0,15 \\
$\mathrm{C}$ & $150-230$ & 0,22 & & & \\
\hline
\end{tabular}

Podzólico Vermelho-Amarelo - var. Laras

Perfil n. ${ }^{\circ} 6$

Perfil n. ${ }^{\circ} 7$

$\begin{array}{cccccc}\text { Horizonte } & \text { Espessura } & \mathrm{K} & \text { Horizonte } & \text { Espessura } & \mathrm{K} \\ \mathrm{A}_{1} & 0-25 & 0,53 & \mathrm{Ap} & 0-60 & 0,52 \\ \mathrm{~A}_{3} & 25-50 & 0,49 & \mathrm{~B}_{2} & 60-120 & 0,50 \\ \mathrm{~B}_{2} & 50-75 & 0,45 & \mathrm{~B}_{3} & 120-150+ & 0,57 \\ \mathrm{~B}_{22} & 75-130 & 0,50 & & & \\ \mathrm{~B}_{23} & 130-210 & 0,50 & & & \\ \mathrm{~B}_{3} & 210+ & 0,50 & & & \end{array}$

\begin{tabular}{cccccc} 
& Perfil n..$^{\circ}$ & \multicolumn{4}{c}{ Perfil n..$^{\circ}$} \\
Horizonte & Espessura & $\mathrm{K}$ & Horizonte & Espessura & $\mathrm{K}$ \\
$\mathrm{Ap}$ & $0-46$ & 0,57 & $\mathrm{~A}_{1}$ & $0-20$ & 0,52 \\
$\mathrm{~B}_{2}$ & $46-100$ & 0,42 & $\mathrm{~B}_{1}$ & $20-40$ & 0,50 \\
$\mathrm{~B}_{3}$ & $100-145$ & 0,43 & $\mathrm{~B}_{2}$ & $40-85$ & 0,52 \\
$\mathrm{C}$ & $145+$ & 0,62 & $\mathrm{~B}_{3}$ & $85-105$ & 0,53 \\
& & & $\mathrm{C}$ & $105+$ & 0,54
\end{tabular}




\begin{tabular}{ccc} 
& Perfil n. $^{\circ} 10$ & \\
Horizonte & Espessura & $\mathrm{K}$ \\
$\mathrm{A}_{1}$ & $0-23$ & 0,29 \\
$\mathrm{~A}_{2}$ & $23-59$ & 0,53 \\
$\mathrm{~B}_{1}$ & $59-77$ & 0,48 \\
$\mathrm{~B}_{22}$ & $77-110$ & 0,42 \\
$\mathrm{~B}_{23}$ & $110-145$ & 0,47 \\
$\mathrm{C}$ & $154+$ & 0,62 \\
\hline
\end{tabular}

Podzólico Vermelho-Amarelo - Intergrade Para Latosol Vermelho-Amarelo Perfil n. ${ }^{\circ} 11$ Perfil n. ${ }^{\circ} 12$

\begin{tabular}{cccccc} 
Horizonte & Espessura & $\mathrm{K}$ & Horizonte & Espessura & $\mathrm{K}$ \\
$\mathrm{A}_{1}$ & $0-20$ & 0,22 & $\mathrm{~A}_{1}$ & $0-18$ & 0,23 \\
$\mathrm{~A}_{3}$ & $20-50$ & 0,26 & $\mathrm{~A}_{3}$ & $18-50$ & 0,25 \\
$\mathrm{~B}_{2} .$. & $50-160$ & 0,14 & $\mathrm{~B}_{1}$ & $50-100$ & 0,12 \\
$\mathrm{~B}_{3}$ & $160-290$ & 0,15 & $\mathrm{~B}_{2}$ & $100-300$ & 0,13 \\
\hline
\end{tabular}

Solios Podzolizados Com Cascalhos

Perfil n..$^{\circ} 13$

Horizonte Espessura K

$\begin{array}{lll}\mathrm{A}_{1} & 0-20 & 0,22 \\ \mathrm{~A}_{2} & 20-40 & 0,38 \\ \mathrm{~B}_{1} & 40-55 & 0,31 \\ \mathrm{~B}_{2} & 55-90 & 0,23 \\ \mathrm{C} & 90-130 & 0,38\end{array}$

Perfil n..$^{\circ} 15$

Horizonte

$A_{1}$

$\mathrm{B}_{1}$

$\mathrm{B}_{22}$

$\mathrm{B}_{3}$

C
Espessura

0-20

20-59

59-117

$117-155$

$155-170+$
0,02

0,11

0,14

0,28
Perfil n. ${ }^{\circ} 14$

$\begin{array}{ccc}\text { Horizonte } & \text { Espessura } & \mathrm{K} \\ \mathrm{A}_{1} & 0-15 & 0,20 \\ \mathrm{~A}_{2} & 15-30 & 0,31 \\ \mathrm{~B}_{2} & 30-90 & 0,20 \\ \mathrm{~B}_{3} & 90-130 & 0,29 \\ \mathrm{C} & 130-150+ & 0,49\end{array}$

Perfil n. ${ }^{\circ} 16$

K Horizonte Espessura K
$0-10$

$10-20$

$20-55$

$55-80$

80-100
$A_{1}$

$B_{21}$

$\mathrm{B}_{22}$

$\mathrm{B}_{3}$

C
0,18

0,13

0,13

0,18

0,32 
Solos Podzolizados de Lins e Marília - var. Lins

\begin{tabular}{cccccc} 
& Perfil n..$^{\circ} 18$ & \multicolumn{3}{c}{ Perfil n..$^{\circ} 19$} \\
Horizonte & Espessura & $\mathrm{K}$ & Horizonte & Espessura & $\mathrm{K}$ \\
$\mathrm{A}_{1}$ & $0-15$ & 0,36 & $\mathrm{~A}_{1}$ & $0-10$ & 0,44 \\
$\mathrm{~A}_{2}$ & $15-30$ & 0,52 & $\mathrm{~A}_{2}$ & $10-35$ & 0,48 \\
$\mathrm{~B}_{21}$ & $30-50$ & 0,50 & $\mathrm{~B}_{21}$ & $35-50$ & 0,55 \\
$\mathrm{~B}_{22}$ & $50-70$ & 0,49 & $\mathrm{~B}_{22}$ & $50-105$ & 0,37 \\
$\mathrm{~B}_{3}$ & $70-200$ & 0,49 & $\mathrm{~B}_{23}$ & $105-170$ & 0,38 \\
& & & & $170+$ & 0,42 \\
& & & & & \\
Horizonte & Espessura & $\mathrm{K}$ & & & \\
Ap & $0-30$ & 0,54 & & & \\
$\mathrm{~B}_{1}$ & $30-50$ & 0,38 & & & \\
$\mathrm{~B}_{21}$ & $50-70$ & 0,34 & & & \\
$\mathrm{~B}_{22}$ & $70-100$ & 0,34 & & & \\
$\mathrm{~B}_{3}$ & $100-150$ & 0,44 & & & \\
\hline
\end{tabular}

Solos Podzolizados de Lins e Marilia - var. Marília

\begin{tabular}{cccccc} 
& Perfil n..$^{\circ} 21$ & \multicolumn{3}{c}{ Perfil n..$^{\circ} 22$} \\
Horizonte & Espessura & $\mathrm{K}$ & Horizonte & Espessura & $\mathrm{K}$ \\
$\mathrm{Ap}$ & $0-21$ & 0,56 & $\mathrm{Ap}$ & $\mathrm{Ap}$ & 0,55 \\
$\mathrm{~B}_{21}$ & $21-40$ & 0,52 & $\mathrm{~A}_{2}$ & $\mathrm{~A}_{2}$ & 0,59 \\
$\mathrm{~B}_{22}$ & $40-85$ & 0,43 & $\mathrm{~B}_{22}$ & $\mathrm{~B}_{22}$ & 0,41 \\
$\mathrm{~B}_{3}$ & $85-197$ & 0,46 & $\mathrm{~B}_{23}$ & $\mathrm{~B}_{3}$ & 0,44 \\
$\mathrm{C}$ & $197-253$ & 0,56 & $\mathrm{~B}_{3}$ & $\mathrm{~B}_{3}$ & 0,49
\end{tabular}

Perfil n..$^{\circ} 23$

Horizonte Espessura $\mathbf{K}$

$\begin{array}{lcc}\mathrm{A}_{1} & 0-10 & 0,32 \\ \mathrm{~A}_{2} & 10-70 & 0,65 \\ \mathrm{~B}_{2} & 70-140 & 0,45 \\ \mathrm{~B}_{3} & 140-170 & 0,49\end{array}$


Mediterrânico Vermielha-Amarelo

Perfil n. ${ }^{0} 27$

Horizonte

$A_{1}$

$\mathrm{A}_{3}$

$\mathrm{B}_{22}$

$\mathrm{B}_{23}$

$\mathrm{B}_{3}$

C
Espessura

0-14

14-33

33-75

75-130

$130-180$

$180-230$
Perfil n. ${ }^{0} 28$

Horizonte Espessura K

Ap $\quad 0-20 \quad 0,16$

$\mathrm{B}_{1} \quad 20-40 \quad 0,23$

$\begin{array}{lll}B_{21} & 40-70 & 0,12\end{array}$

$\begin{array}{lll}\mathrm{B}_{22} & 70-100 & 0,14\end{array}$

$\mathrm{B}_{22} \quad 100-135 \quad 0,16$

$\begin{array}{lll}\mathrm{B}_{3} & 135-250 & 0,18\end{array}$

Terra Roxa Estruturada

\begin{tabular}{cccccc} 
& Perfil n. ${ }^{0} 29$ & \multicolumn{4}{c}{ Perfil n. ${ }^{\circ} 30$} \\
Horizonte & Espessura & $\mathrm{K}$ & Horizonte & Espessura & $\mathrm{K}$ \\
$\mathrm{Ap}$ & $0-19$ & 0,03 & $\mathrm{~A}_{1}$ & $0-20$ & 0,02 \\
$\mathrm{~B}_{22}$ & $19-80$ & 0,06 & $\mathrm{~B}_{1}$ & $20-45$ & 0,07 \\
$\mathrm{~B}_{23}$ & $80-134$ & 0,09 & $\mathrm{~B}_{22}$ & $45-80$ & 0,07 \\
$\mathrm{~B}_{3}$ & $134-224$ & 0,12 & $\mathrm{~B}_{23}$ & $80-110$ & 0,08 \\
$\mathrm{C}$ & $224-250$ & 0,28 & $\mathrm{~B}_{3}$ & $110-150$ & 0,09 \\
& & & & & \\
& Perfil n. ${ }^{0} 31$ & & & & \\
Horizonte & Espessura & $\mathrm{K}$ & & & \\
$\mathrm{Ap}$ & $0-10$ & 0,04 & & & \\
$\mathrm{~B}_{1}$ & $10-28$ & 0,12 & & & \\
$\mathrm{~B}_{22}$ & $28-132$ & 0,08 & & & \\
$\mathrm{~B}_{23}$ & $132-223$ & 0,15 & & & \\
\hline
\end{tabular}

\section{Latossol Roxo}

\begin{tabular}{cccccc} 
& Perfil n..$^{\circ} 33$ & \multicolumn{4}{c}{ Perfil n. 34} \\
Horizonte & Espessura & $\mathrm{K}$ & Horizonte & Espessura & $\mathrm{K}$ \\
$\mathrm{Ap}$ & $\mathbf{0 - 8}$ & 0,06 & $\mathrm{Ap}_{\mathrm{p}}$ & $0-15$ & 0,04 \\
$\mathrm{~A}_{3}$ & $8-23$ & 0,06 & $\mathrm{~A}_{3}$ & $15-45$ & 0,09 \\
$\mathrm{~B}_{1}$ & $23-100$ & 0,11 & $\mathrm{~B}_{21}$ & $45-90$ & 0,12 \\
$\mathrm{~B}_{22}$ & $100-200$ & 0,15 & $\mathrm{~B}_{22}$ & $90-130$ & 0,13
\end{tabular}


Perfil n. ${ }^{\circ} 35$

Perfil n. ${ }^{\circ} 36$

\begin{tabular}{cccccc} 
Horizonte & Espessura & $\mathrm{K}$ & Horizonte & Espessura & $\mathrm{K}$ \\
$\mathrm{A}_{1}$ & $0-25$ & 0,06 & $\mathrm{~A}_{1}$ & $0-40$ & $\mathbf{0 , 0 4}$ \\
$\mathrm{A}_{3}$ & $25-45$ & 0,05 & $\mathrm{~A}_{3}$ & $40-65$ & 0,05 \\
$\mathrm{~B}_{21}$ & $45-95$ & 0,09 & $\mathrm{~B}_{21}$ & $65-90$ & 0,09 \\
$\mathrm{~B}_{22}$ & $95-120$ & 0,14 & $\mathrm{~B}_{22}$ & $\mathbf{9 0 - 1 2 0}$ & $\mathbf{0 , 1 1}$ \\
\hline
\end{tabular}

Latosol Vermelho-Escuro - Orto

\begin{tabular}{cccccc} 
& Perfil n..$^{\circ} 40$ & \multicolumn{3}{c}{ Perfil n. $^{\circ} 41$} \\
Horizonte & Espessura & $\mathrm{K}$ & Horizonte & Espessura & $\mathrm{K}$ \\
$\mathrm{A}_{1}$ & $0-3$ & 0,04 & $\mathrm{~A}_{1}$ & $0-20$ & 0,16 \\
$\mathrm{~A}_{3}$ & $3-36$ & 0,09 & $\mathrm{~B}_{1}$ & $20-50$ & 0,13 \\
$\mathrm{~B}_{1}$ & $36-60$ & 0,11 & $\mathrm{~B}_{21}$ & $50-120$ & 0,12 \\
$\mathrm{~B}_{21}$ & $60-120$ & 0,10 & $\mathrm{~B}_{22}$ & $120-180$ & 0,13 \\
$\mathrm{~B}_{22}$ & $120-170$ & 0,12 & & &
\end{tabular}

Perfil n. ${ }^{\circ} 42$

\begin{tabular}{ccc} 
Horizonte & Espessura & $\mathrm{K}$ \\
$\mathrm{A}_{1}$ & $0-30$ & 0,03 \\
$\mathrm{~B}_{1}$ & $30-60$ & 0,05 \\
$\mathrm{~B}_{21}$ & $60-150$ & 0,05 \\
$\mathrm{~B}_{22}$ & $150-210$ & 0,06 \\
\hline
\end{tabular}

Latosol Vermelho-Escuro - Fase Arenosa

\begin{tabular}{cccccc} 
& Perfil n..$^{\circ} 43$ & \multicolumn{3}{c}{ Perfil n. $^{\circ} 44$} \\
Horizonte & Espessura & $\mathrm{K}$ & Horizonte & Espessura & $\mathrm{K}$ \\
$\mathrm{A}_{1}$ & $0-23$ & 0,39 & $\mathrm{~A}_{1}$ & $0-15$ & 0,35 \\
$\mathrm{~B}_{1}$ & $23-90$ & 0,44 & $\mathrm{~B}_{1}$ & $15-35$ & 0,44 \\
$\mathrm{~B}_{21}$ & $90-145$ & 0,44 & $\mathrm{~B}_{21}$ & $35-60$ & 0,49 \\
$\mathrm{~B}_{22}$ & $145-275$ & 0,44 & $\mathrm{~B}_{22}$ & $60-120$ & 0,49
\end{tabular}

\begin{tabular}{cccccc} 
& Perfil n..$^{\circ} 45$ & \multicolumn{3}{c}{ Perfil n..$^{\circ} 46$} \\
Horizonte & Espessura & $\mathrm{K}$ & Horizonte & Espessura & $\mathrm{K}$ \\
$\mathrm{Ap}$ & $0-25$ & 0,29 & $\mathrm{~A}_{1}$ & $0-20$ & 0,27 \\
$\mathrm{~B}_{1}$ & $25-55$ & 0,29 & $\mathrm{~B}_{1}$ & $20-50$ & 0,38 \\
$\mathrm{~B}_{21}$ & $55-95$ & 0,34 & $\mathrm{~B}_{21}$ & $50-100$ & 0,38 \\
$\mathrm{~B}_{22}$ & $95-200$ & 0,35 & $\mathrm{~B}_{22}$ & $100-200$ & $\mathbf{0 , 3 8}$ \\
\hline
\end{tabular}


Lafosol Vermelho-Amarelo - Orto

Perfil n. ${ }^{\circ} 47$

Horizonte

$\mathrm{Ap}$
$\mathrm{A}_{3}$
$\mathrm{~B}_{1}$
$\mathrm{~B}_{21}$
$\mathrm{~B}_{22}$

Espessura

$0-8$

8-28

28-94

94-130

$130-220$

Perfil n. 049

Horizonte
$A_{1}$
$B_{11}$
$\mathrm{B}_{12}$
$\mathrm{B}_{21}$

Espessura

0-18

$18-65$

$65-100$

$100-200$

\section{Perfil n. ${ }^{\circ} 48$}

$\begin{array}{ccc}\text { Horizonte } & \text { Espessura } & \mathrm{K} \\ \mathrm{A}_{1} & 0-10 & 0,10 \\ \mathrm{~A}_{3} & 10-35 & 0,12 \\ \mathrm{~B}_{1} & 35-100 & 0,12 \\ \mathrm{~B}_{2} & 100-200 & 0,13\end{array}$

Latosol Vermelho-Amarelo - Fase Rasa

Perfil n. ${ }^{\circ} 50$

\begin{tabular}{cccccc} 
Horizonte & Espessura & $\mathrm{K}$ & Horizonte & Espessura & $\mathrm{K}$ \\
$\mathrm{A}_{1}$ & $0-12$ & 0,17 & $\mathrm{~A}_{1}$ & $0-10$ & 0,10 \\
$\mathrm{~B}_{1}$ & $12-45$ & 0,27 & $\mathrm{~A}_{3}$ & $10-40$ & 0,16 \\
$\mathrm{~B}_{2}$ & $45-103$ & 0,20 & $\mathrm{~B}_{2}$ & $40-90$ & 0,20 \\
$\mathrm{~B}_{3}$ & $103-140$ & 0,30 & & & \\
\hline
\end{tabular}

\section{Latosol Vermelho-Amarelo - Fase Arenosa}

Perfil n. ${ }^{\circ} 52$

Horizonte
$A_{1}$
$A_{3}$
$B_{1}$
$\mathrm{B}_{22}$

Espessura

0-30

30-64

64-166

166-391

Perfil n. ${ }^{\circ} 55$

Horizonte

$A_{1}$
$A_{3}$
$B_{1}$
$B_{2}$

$A_{1}$

$B_{1}$

$\mathrm{B}_{2}$

Espessura
$\mathrm{K}$

0,22

0,25

0,25

0,27

Perfil n. ${ }^{\circ} 53$

Horizonte Espessura $\mathrm{K}$

$\begin{array}{ccc}A_{1} & 0-15 & 0,08 \\ A_{3} & 15-35 & 0,09 \\ B_{1} & 35-70 & 0,15 \\ B_{2} & 270-200 & 0,15\end{array}$

Perfil n. ${ }^{\circ} 56$

K

Horizonte

Espessura

K

0,12

0,19

0,16

0,19

$0-40$

40-70

70-120

$120-200$
0,37

0,32

0,32

0,34 
Latosol Vermelho-Amarelo - Fase Terraço

\begin{tabular}{cccccc} 
& Perfil n..$^{0} 58$ & \multicolumn{3}{c}{ Perfil n. $^{0} 59$} \\
Horizonte & Espessura & $\mathrm{K}$ & Horizonte & Espessura & $\mathrm{K}$ \\
$\mathrm{A}_{11}$ & $0-35$ & $\mathbf{0 , 0 3}$ & $\mathrm{A}_{1}$ & $0-7$ & 0,06 \\
$\mathrm{~A}_{12}$ & $35-80$ & $\mathbf{0 , 0 5}$ & $\mathrm{A}_{3}$ & $7-36$ & 0,12 \\
$\mathrm{~A}_{3}$ & $80-100$ & 0,09 & $\mathrm{~B}_{1}$ & $36-135$ & 0,13 \\
$\mathrm{~B}_{1}$ & $100-120$ & 0,12 & $\mathrm{~B}_{2}$ & $135-315$ & 0,15 \\
$\mathrm{~B}_{2}$ & $120-260$ & $\mathbf{0 , 1 3}$ & & & \\
\hline
\end{tabular}

Perfil n. ${ }^{\circ} 63$

\begin{tabular}{ccc} 
Horizonte & Espessura & $\mathrm{K}$ \\
$\mathrm{A}_{11}$ & $0-12$ & 0,12 \\
$\mathrm{~A}_{12}$ & $12-27$ & 0,15 \\
$\mathrm{~A}_{31}$ & $27-47$ & 0,13 \\
$\mathrm{~A}_{32}$ & $47-136$ & 0,17 \\
$\mathrm{~B}_{1}$ & $136-216$ & 0,14 \\
\hline
\end{tabular}

Latosol Vermelho-Amarelo - Infergrade Para Podzólico Vermelho-Amarelo Perfil n. ${ }^{\circ} 64$ Perfil n. ${ }^{\circ} 65$

$\begin{array}{cccccc}\text { Horizonte } & \text { Espessura } & \mathrm{K} & \text { Horizonte } & \text { Espessura } & \mathrm{K} \\ \mathrm{A}_{1} & 0-25 & 0,04 & \mathrm{~A}_{1} & 0-8 & 0,09 \\ \mathrm{~A}_{3} & 25-40 & 0,12 & \mathrm{~A}_{3} & 8-30 & 0,10 \\ \mathrm{~B}_{1} & 40-60 & 0,18 & \mathrm{~B}_{21} & 30-90 & 0,10 \\ \mathrm{~B}_{21} & 60-90 & 0,20 & \mathrm{~B}_{22} & 90-180 & 0,11 \\ \mathrm{~B}_{22} & 90-120 & 0,24 & & & \\ \mathrm{~B}_{3} & 120-160 & 0,32 & & & \end{array}$

Perfil n. ${ }^{\circ} 66$

$\begin{array}{ccc}\text { Horizonte } & \text { Espessura } & \mathrm{K} \\ \mathrm{A}_{1} & 0-13 & 0,03 \\ \mathrm{~A}_{3} & 13-25 & 0,04 \\ \mathrm{~B}_{1} & 25-40 & 0,17 \\ \mathrm{BB}_{21} & 40-65 & 0,12 \\ \mathrm{~B}_{22} & 65-120 & 0,11 \\ \mathrm{~B}_{3} & 120-180 & 0,15\end{array}$


Latosol Vermelho-Amarelo Húmico

Perfil n. ${ }^{\circ} 67$

Horizonte

$\mathrm{A}_{11}$
$\mathrm{~A}_{12}$
$\mathrm{~A}_{13}$
$\mathrm{~A}_{3}$
$\mathrm{~B}_{1}$

Espessura

$0-12$

$12-50$

50-95

95-155 155-243
Perfil n. ${ }^{\circ} 68$

K Horizonte Espessura K
$A_{11}$

$A_{12}$

$A_{13}$

$\mathrm{A}_{3}$
0-30

$30-85$

85-185

$185-240$
0,04

0,07

0,10

0,12

0,13

Solos de Campos do Jordão

$$
\text { Perfil n. }{ }^{\circ} 70
$$

\section{Perfil n. ${ }^{\circ} 71$}

\begin{tabular}{cccccc} 
Horizonte & Espessura & $\mathrm{K}$ & Horizonte & Espessura & $\mathrm{K}$ \\
$\mathrm{A}_{1}$ & $0-70$ & 0,12 & $\mathrm{~A}_{1}$ & $0-10$ & 0,10 \\
$\mathrm{~B}_{1}$ & $70-85$ & 0,15 & $\mathrm{~B}_{1}$ & $10-40$ & 0,15 \\
$\mathrm{~B}_{21}$ & $85-120$ & 0,16 & $\mathrm{~B}_{2}$ & $40-65$ & 0,17 \\
$\mathrm{~B}_{22}$ & $120-195$ & 0,16 & $\mathrm{C}_{1}$ & $65-110$ & 0,44 \\
& & & $\mathrm{C}_{2}$ & $110-160$ & 0,67 \\
\hline
\end{tabular}

\section{Podzol Hidromórfico}

$$
\text { Perfil n. }{ }^{\circ} 74
$$

Horizonte Espessura K

$\begin{array}{lcc}\mathrm{A}_{1} & 0-25 & 0,46 \\ \mathrm{~A}_{2} & 25-50 & 0,62 \\ \mathrm{~B}_{2^{\mathrm{h}}} & 50-90 & 0,55 \\ \mathrm{~B}_{\text {2hir }} & 90-91 & 0,49 \\ \mathrm{C} & 91-330 & 0,55\end{array}$

\begin{tabular}{ccc} 
Litosol - Fase Substrato & Basaltico \\
& Perfil n. ${ }^{\circ} 76$ \\
Horizonte & Espessura & $\mathrm{K}$ \\
$\mathrm{A}_{1}$ & $0-30$ & 0,12 \\
(B) & $30-35$ & 0,35 \\
$\mathrm{R}$ & $35-200+$ & 0,32 \\
\hline
\end{tabular}




\section{Litosol Fase Substrato Granito-Gnaisse}

$$
\text { Perfil n. }{ }^{0} 77
$$

Horizonte Espessura K

$\begin{array}{lcc}A_{1} & 0-22 & 0,13 \\ (B) & 22-31 & 0,26 \\ R & 31-50+ & 0,38\end{array}$

\begin{tabular}{ccc} 
Litosol Fase & Filito-Xisto & \\
& Perfiln. ${ }^{\circ} 78$ & \\
Horizonte & Espessura & $\mathrm{K}$ \\
$\mathrm{A}_{1}$ & $0-19$ & 0,24 \\
$\mathrm{~B} / \mathrm{C}$ & $19-23$ & 0,52 \\
$\mathrm{R}$ & $23-110$ & 0,67 \\
\hline
\end{tabular}

\section{Litosol Fase Substrato Arenito Calcário}

Perfil n. ${ }^{\circ} 80$

$\begin{array}{ccc}\text { Horizonte } & \text { Espessura } & \mathrm{K} \\ \mathrm{A}_{1} & 0-15 & 0,43 \\ \mathrm{~A}_{2} & 15-25 & 0,52 \\ \mathbf{R} & 25+ & -\end{array}$

\section{Litosol - Fase-Substrato Folhelho Argilito}

\begin{tabular}{ccc}
\multicolumn{2}{c}{ Perfil } & - Levantamento \\
Horizonte & Espessura & $\mathrm{K}$ \\
Ap & $0-30$ & 0,24 \\
C/R & $30-65+$ & 0,49
\end{tabular}

\section{Regosol}

\begin{tabular}{cccccc} 
& Perfil n..$^{\circ} 83$ & \multicolumn{3}{c}{ Perfil n..$^{\circ} 84$} \\
Horizonte & Espessura & $\mathrm{K}$ & Horizonte & Espessura & $\mathrm{K}$ \\
$\mathrm{A}_{11}$ & $0-15$ & 0,40 & $\mathrm{~A}_{1}$ & $0-15$ & 0,28 \\
$\mathrm{~A}_{12}$ & $15-120$ & 0,43 & $\mathrm{~A}_{3}$ & $15-60$ & 0,38 \\
$\mathrm{~A}_{3}$ & $120-190$ & 0,52 & $(\mathrm{~B})$ & $60-200$ & 0,37 \\
\hline
\end{tabular}


Regosol (Intergrade Para L. V. A.) e ((Intergrade Para P. V. A.) D Perfil n..$^{\circ} 85$ Perfil n. ${ }^{\circ} 86$

\begin{tabular}{ccccrc} 
Horizonte & Espessura & $\mathrm{K}$ & Horizonte & Espessura & $\mathrm{K}$ \\
$\mathrm{A}_{1}$ & $0-15$ & 0,44 & $\mathrm{~A}_{1}$ & $0-15$ & 0,43 \\
$\mathrm{~A}_{3}$ & $15-49$ & 0,45 & $\mathrm{~B}_{1}$ & $15-35$ & 0,46 \\
$\mathrm{~B}_{1}$ & $49-112$ & 0,52 & $\mathrm{~B}_{21}$ & $35-70$ & 0,43 \\
$\mathrm{~B}_{21}$ & $112-148$ & 0,52 & $\mathrm{~B}_{22}$ & $70-140$ & 0,44 \\
$\mathrm{~B}_{22}$ & $148-328$ & 0,52 & $\mathrm{~B}_{3}$ & $140-200$ & 0,46 \\
\hline
\end{tabular}

\section{Hidromórfico}

Perfil - Levantamento dúe Solos do Município de Piracicaba Horizonte Espessura $\mathrm{K}$

\begin{tabular}{lrl}
$\mathrm{A}_{1}$ & $0-15$ & 0,17 \\
$\mathrm{~B}_{28}$ & $15-40$ & 0,17 \\
$\mathrm{C}_{8}$ & $40-80$ & 0,18 \\
\hline
\end{tabular}

